\title{
Ultrasonic Interferometer for High-Accuracy Linear Measurements
}

\author{
E. Konkov \\ Russian Academy of Sciences, Institute of Radioengineering and Electronics, Fryazino Department (FIRE RAS), \\ Vvedenskogo sq.1, Fryazino, Moscow region, 141190, Russia, e-mail: ekonkov@yandex.ru
}

\begin{abstract}
An application of ultrasonic interferometry is proposed and investigated as a new possible technique for high-accuracy linear and angular measurements. The basic principle of the proposed technique is the use of coherent running acoustic wave propagation along homogeneous solid waveguide in the capacity of linear measuring scale. The RMS sensitivity to the linear drift of $0.5 \mu \mathrm{m}$ and RMS error of absolute measurement of $\pm 12 \mu \mathrm{m}$ over the distance up to one meter have been achieved experimentally.
\end{abstract}

Keywords: ultrasound, interferometer, linear measurements, quartz fiber

\section{INTRODUCTION}

$\mathrm{M}$ ODERN fast development of high-precision machinery construction, robotics and other branches of technology demands an adequate progress on spatial metrology of machines' fast-moving parts. The accuracy of measurements required is $10^{-6}$ while motion velocity of mechanical parts runs up to several meters per second.

Any solution of this problem is not possible without application of some new physical and systematic principles.

A subject of this investigation is the possibility to use acoustic continuous wave process for high-precision absolute spatial measurements performing with high speed.

\section{BASIC PRINCIPLE OF THE SYSTEM}

Main idea of the technique proposed is the use of coherent running acoustic wave propagation along homogeneous solid waveguide as a linear measuring scale. That is, a special kind of ultrasonic inteferometry is proposed as an application for high-precision linear and (or) angular measurement.

An obvious advantage of coherent running wave interferometers as opposed to any pulse system is the possibility to provide high-accurate phase measurements with averaging over a lot of wave periods.

The proposed principle is very close to optical interferometry, but it has several key advantages.

First: most optical interferometric measurements are being performed in air. Index of air refraction spatial and thermal instability is limiting the accuracy by the value of $10^{-5}$ in any case. To perform these measurements in vacuum is not possible. Any solid waveguide is much more stable, but solid waveguides can be used only with the acoustic variant.

Second: because optical waves are very short (about $1 \mu \mathrm{m})$ in comparison to acoustic ones, it is hardly possible to carry out optical interferometric measurement of fast-changing distance. In this case the repetition rate of interferential maximums is rather high for reliable acquisition and exact processing even for any modern electronic system. Acoustic systems are free of these problems.

Third: acoustic elements and systems are much more simple and cheaper than optical ones.

\section{EXPERIMENTAL SETUP}

On the base of this proposed technique an experimental setup has been developed. The simplified block-diagram of this system is shown in Fig. 1 bellow.

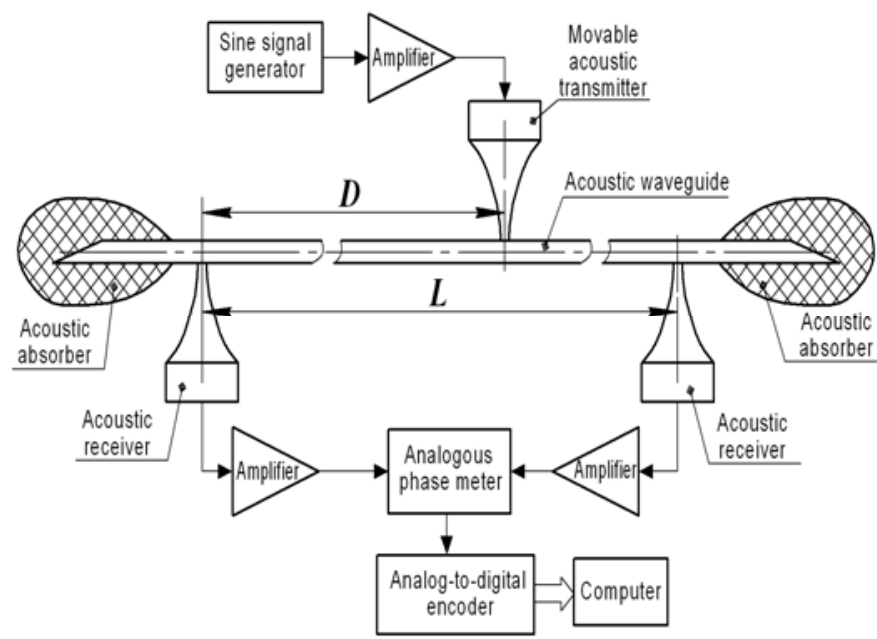

Fig.1 Ultrasonic interferometric encoder of distance.

The simplified block-diagram.

$\boldsymbol{D}$ - the distance to be measured,

$\boldsymbol{L}$ - base of the interferometer, the distance between receivers.

In fact the setup created is a breadboard model of the linear ultrasonic transducer (encoder), where the waveguide system constitutes of a core of melted quartz and piezoelectric converters providing a radiation and receipt of continuous coherent acoustic wave.

Design and material of ultrasonic waveguide are principal items of the system. The waveguide is made of a piece of optic fiber. The fiber material is melted quartz of F500 type having a coefficient of thermal expansion better than the same one of super invar that is less than $10^{-6} /{ }^{\circ} \mathrm{C}$ and consequently, it has a thermal sonic speed dependence of the same order. Therewith its acoustical absorption is negligibly small. The value of the absorption measured is about $1 \mathrm{~dB} / \mathrm{m}$. 
Furthermore, modern fiber-optic technology provides the highest homogeneity of physical and geometrical waveguide parameters along its length. Thus, this waveguide structure is able to provide the linear absolute measurements with relative error of $10^{-6}$.

Both terminals of waveguide have acoustic absorbers made of special soft rubber to eliminate reflections and to provide a regime of running wave without interference.

Total length of the waveguide used is $1 \mathrm{~m}$.

Piezoelectric units (transmitter and receivers) are made of piezoceramics working in resonant regime. Each piezoelectric converter is supplied with special exponential ultrasonic concentrator designed especially for optimal matching between piezoceramic elements and waveguide.

Transmitter unit is mounted on a movable part of the system and the linear position of this unit along the waveguide axis is a value to be measured. The frequency stability of the radiating ultrasound is better than $10^{-8}$, it is provided by a temperature-controlled quartz-crystal oscillator.

Two receiving units are mounted on opposite terminals of the waveguide, so the phase difference between electrical sinusoidal signals from these two receivers is quite linear by the distance to be measured: $D=\Delta \varphi \lambda / \mathbf{4 \pi}$, where $D$ is a distance being measured, $\Delta \varphi-$ phase difference, $\lambda$ is sonic wavelength in the waveguide.

Thus, the described device is in fact an ultrasonic interferometer.

The phase difference between sine signals from receivers is measured by phase meter with an analogous output. Then this voltage is digitized and fed into a computer for following acquisition and processing.

\section{RESUltS AND CONCLUSIONS}

In various performed experiments the waveguide diameter and wavelength in single mode regime have been varied at the limits of $0.5-1.5 \mathrm{~mm}$ and $1-2.5 \mathrm{~mm}$ correspondingly.

For metrological testing of the system over the distances less than $80 \mathrm{~mm}$ the movable metric part of a measuring microscope with an accuracy of $1 \mu \mathrm{m}$ was used. For the testing up to $1 \mathrm{~m}$ an optical raster transducer of $4 \mu \mathrm{m}$ accuracy was used as reference gage [1].

A typical derivation chart of distance measured by the tested system in relation to the reference gage is shown in Fig.2.

The RMS sensitivity to the linear drift of $0.5 \mu \mathrm{m}$ has been achieved. It is much better than the same value of impact ultrasonic magnetostrictive linear transducers developed by Balluff [2] or Gefran [3] companies, for example. The RMS error of absolute measurement is $\pm 12 \mu \mathrm{m}$ over the distance up to one meter. The maximum speed of measured distance variation is calculated to be $10 \mathrm{~m} / \mathrm{sec}$.

But unlike Balluff's and Gefran's transducers the interferometer created by us has an applied imperfection due to the contact mode of ultrasound inlet into waveguide. This imperfection should be eliminated by using some kind of non- contact transmitters. It could be magnetostrictive or optoacoustical generation of sound, for example. In any case the development of non-contact acoustic inlet system for quartz fiber waveguide is a serious separate problem to be solved. So, we intend to continue our research in this direction.

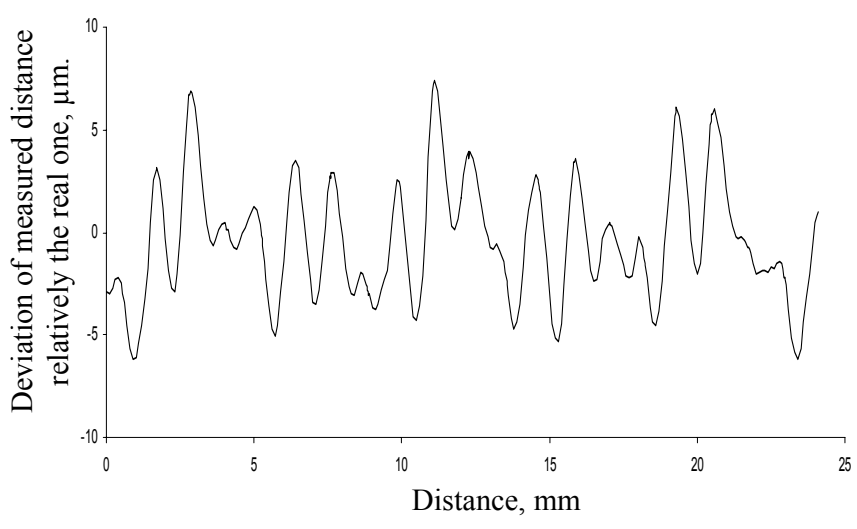

Fig.2 A typical deviation chart of measured distance in relation to the real one.

The parameters achieved experimentally are not a theoretical limit for this technique. There are reserves for essential improvement of the achieved system accuracy.

Also it is possible to develop an angular absolute transducer using the technique proposed if the basic acoustic waveguide is performed by circular configuration. R\&D of the ultrasonic angular transducer is the next step of the presented researches.

\section{ACKNOWLEDGMENT}

We would like to thank SKB IS Company for the possibility to use their equipment for the experiment performed and also for essential financial support.

Special appreciations to Mr. Polyakov the chief engineer of SKB IS.

\section{REFERENCES}

[1] SKB IS (2004). Transducers of Linear Displacement. Catalog and Brief Products Descriptions. Retrieved from http://www.skbis.ru (in Russian language)

[2] BALLUFF, Sensors Worldwide (2006-2008). Balluff Micropulse Liner Position Transducers. Retrieved from http://www.balluff.com/Balluff/us/ProductsChannel/Ov erview/en/Micropulse+Transducers.htm

[3] GEFRAN (2006). The Updated Series of Inside Cylinder Magnetostrictive Transducers. Retrieved from http://www.gefran. com /productnews_dettaglio .aspx? ID $=977$ 